\title{
THE ACCURACY OF THE ICRF: AN INTERCOMPARISON OF VLBI ANALYSIS SOFTWARE
}

\author{
C.S. JACOBS AND O.J. SOVERS \\ Jet Propulsion Laboratory (JPL) \\ 4800 Oak Grove Dr., Pasadena, CA 91109-8099 \\ D. GORDON AND C. MA \\ Goddard Space Flight Center (GSFC) \\ Code 926, Greenbelt, $M D$, 20771 \\ AND \\ A.-M. GONTIER \\ Observatoire de Paris \\ 61 Avenue de l'Observatoire, F-75014 Paris France
}

\section{Introduction}

As discussed in other papers in this volume, the IAU XXIII General Assembly adopted a new fundamental celestial reference frame: the International Celestial Reference Frame (ICRF) based on VLBI observations of extragalactic radio sources (Ma et al., 1997). It is approximately 300 times more accurate than its predecessor, the FK5. At present, no other technique has produced a more accurate celestial frame than VLBI. Since no other astrometric technique provides an external standard of accuracy, the VLBI claim of a great leap forward in accuracy must be verified by internal consistency tests. This paper addresses one aspect of internal consistency: the ability of independent VLBI software packages to reproduce a celestial frame without significant loss of accuracy. This is no small task since the software packages are large - involving on the order of 100000 lines of code. What does VLBI software do? Aside from routines designed to collect the data and extract raw observables which will not be considered here, its principal task is to model the differential group delay and phase delay rate of radio signals received at two widely separated antennas (Sovers, Fanselow \& Jacobs, 1998). The software then refines this model via a least squares adjustment of relevant physical parameters which describe station locations, source positions, clock offsets, atmospheric refraction, tidal effects, etc. In the early 1990s, studies revealed that differences in software implementation and analyst's choices of model options were one of the largest contributors to differences in independent calculations of VLBI celestial frames. These differences were of comparable size to the formal uncertainties of the celestial frame's source positions.

\section{Comparisons of VLBI analysis software}

In order to isolate the cause of these differences, we compared three software packages: JPL's MODEST (Sovers, Fanselow \& Jacobs, 1998), GSFC's CALC/SOLVE (Ma, 1978) and Paris Observatory's GLORIA (Gontier, 1992). Because almost all available high quality astrometric VLBI data was already set up for analysis by GSFC, their software was chosen to produce the ICRF. Because of the complexity of the software, the first step taken was to compare the calculations of each VLBI model component from each software package. This comparison was limited to data taken on 27 March 1992 with Deep Space Network antennas in Goldstone, CA and Madrid, Spain, and a second experiment which included a southern hemisphere station: Tidbinbilla, Australia to Goldstone, CA on 22 March 1992. After considerable effort, we reached our goal of $1 \mathrm{ps}$ and $1 \mathrm{fs} / \mathrm{s}$ wrms agreement in delay and delay rate, respectively $(<0.01$ mas). The geometric component (station locations, source positions, relativity) of the delay models agreed to much better than $1 \mathrm{ps}$. 
The solid Earth tide models limited the accuracy to the stated levels. Antenna axis offset and troposphere gradient models were not tested.

\section{Celestial frame comparisons and analyst choices}

Once the model implementations were verified, we constructed a full VLBI reference frame using the GSFC and JPL software (time constraints prevented Paris Observatory from producing a frame). The initial comparison was based on a limited data set of just 11,600 delay/rate observation pairs taken between Aug. 1988 and Feb. 1993 using single baselines from JPL's Deep Space Network. This restricted data set allowed the GSFC and JPL analysts to carefully match a priori model parameters and modeling options. Analyst choices to remove outlier data points were not forced to agree a priori, but, in fact, editing choices differed for only $1 \%$ of the observations (mostly due to an error in the exchange of raw data). The 267 sources in the resulting frames agreed to 0.15 and 0.21 mas wrms in right ascension $(\alpha \cos \delta)$ and declination, $(\delta)$, respectively. These differences were slightly smaller than the formal uncertainties of the individual frames and are thought to be due to differences in the number of parameters used by GSFC and JPL analysts to describe clock and troposphere behavior. There was insufficient time to match exactly the details of these parameterizations. Coordinate biases were 0.01 and 0.02 mas in $\alpha \cos \delta$ and $\delta$, respectively. Rotational alignment was $\leq 0.1$ mas in each of three orthogonal directions. Lastly, we made a frame comparison using a much more extensive data set of $>10^{6}$ delay/rate observation pairs spanning 1978 to 1997 , which included multiple baseline sessions using several global networks of stations. This extensive data set largely overlaps the data set used to construct the ICRF. However, its large size precluded the careful matching of modeling options, data editing, and parameterization choices that were possible with the smaller data set. Despite these limitations, the advantages of averaging over a large number of observations prevailed. The 400 sources common to the resulting GSFC and JPL frames agreed to 0.07 and 0.10 mas wrms in $\alpha \cos \delta$ and $\delta$, respectively. Coordinate biases were $\leq 0.005$ mas and rotational offsets were $\leq 0.08$ mas about all three axes. This agreement is exclusive of the 0.5 mas zonal differences caused by an analyst's choice of whether or not to estimate troposphere gradients (Ma et al., 1997).

\section{Conclusions}

The newly adopted ICRF claims to be accurate to $\approx 0.3$ mas. Such an accuracy requires that the analysis software used to construct the ICRF be free from any errors that couid potentially degrade the frame at a significant level. To that end, we compared JPL's MODEST, GSFC's CALC/SOLVE, and Paris Observatory's GLORIA software in order to verify that they reproduce each other's calculations. Agreement was achieved to a level of $\leq 0.01$ mas thereby ensuring that model implementation is not a significant error. Comparison of reference frames derived from GSFC and JPL VLBI software indicate that analyst modeling option choices, data editing, and model parameterization using VLBI software may contribute 0.1 to 0.2 mas of uncertainty to the newly adopted ICRF. This partially accounts for the 0.25 mas noise floor used in deriving the "realistic" crrors reported for the final ICRF source coordinates.

\section{References}

Gontier, A.-M., (1992) "Orientation de la Terre par Mesure VLBI", Ph. D. dissertation, Observatoire de Paris, Paris, France.

Ma, C., (1978) "VLBI Applied to Polar Motion, Relativity and Geodesy" Ph. D. dissertation, University of Maryland; also NASA TM 79582, GSFC, Greenbelt MD.

Ma, C., Arias, E.F., Eubanks, T.M., Fey, A.L., Gontier, A.-M., Jacobs, C.S., Sovers, O.J., Archinal, B.A., and Charlot, P. (1997) in IERS Technical Note 23, C. Ma and M. Feissel, eds., Observatory of Paris, Paris, France. See also: Ma, C., et al. (1998) "The ICRF Based on VLBI Observations of Extragalactic Radio Sources", in prep. $A \& A$.

Sovers, O. J., Fanselow, J.L., and Jacobs, C.S., (1998) "Astrometry and Geodesy with Radio Interferometry: Experiments, Models, Results", Rev. Mod. Phys., 70 